\title{
Effects of six weeks of resistance-endurance training on microRNA-29 expression in the heart of ovariectomised rats
}

\author{
Morteza Jourkesh ${ }^{1}$, Rahman Soori ${ }^{2}$, Conrad P. Earnest ${ }^{3}$, Lamia Mirheidari ${ }^{4}$, Ali Asghar Ravasi², \\ Stephen R. Stannard ${ }^{5}$, Matias Monsalves-Alvarez ${ }^{6,7}$
}

${ }^{1}$ Department of Physical Education and Sports Science, Shabestar Branch, Islamic Azad University, Iran

${ }^{2}$ Department of Exercise Physiology, Faculty of Physical Education and Sport Sciences, University of Tehran, Tehran, Iran

${ }^{3}$ Department of Health and Kinesiology, Texas A\&M University, College Station, Texas, United States

${ }^{4}$ Department of Exercise Physiology, Faculty of Physical Education and Sport Sciences, University of Tehran,

Aras International Campus, Tehran, Iran

${ }^{5}$ School of Sport, Exercise, and Nutrition, Massey University, Palmerston North, New Zealand

${ }^{6}$ Nutrition and Physical Activity Laboratory, Institute of Nutrition and Food Technology, University of Chile, Santiago, Chile

${ }^{7}$ Department of Biological Sciences, Faculty of Life Sciences, University Andres Bello, Santiago, Chile

\begin{abstract}
Introduction: Heart disease risk rises with age. However, women's symptoms become more pronounced following the onset of menopause. The aim of the present study was to evaluate the effects of six weeks of combined resistance-endurance (RE) training on microRNA-29 expression in the heart of ovariectomised rats.

Material and methods: Thirty female Wistar rats were divided into three groups: 1) sham (SHAM); 2) ovariectomy (OVX); and 3) OVX with RE training (OVX + RE). The effects of these treatments on cardiac microRNA-29 expression were measured using real-time PCR. Data were analysed using a $2 \times 3$ ANOVA and Tukey post-hoc comparisons and presented as mean \pm SEM.

Results: Ovariectomy resulted in a significant down-regulation in the heart microRNA-29 gene expression of OVX ( $0.265 \pm 0.031$ fold changes), OVX $+\mathrm{RE}(0.699 \pm 0.038$ fold changes) in animals vs. sham animals $(1 \pm 0$ fold changes; all, $p<0.05)$ following six weeks of treatment. However, microRNA-29 expression in the OVX $+\mathrm{RE}$ group was significantly greater than in the OVX group $(p<0.05)$.

Conclusions: Our findings suggest that the six weeks of regular RE training attenuate the reduction in heart muscle microRNA-29 expression observed in ovariectomised rates. If our findings carry over to humans, such an exercise regimen could be beneficial to the cardiovascular disease risk in women during menopause.
\end{abstract}

Key words: ovariectomy, exercise training, microRNA-29, cardiac.

\section{Introduction}

Premature menopause, including ovarian surgery, places women at higher all-cause risk. One of the most important causes of death in women who undergo bilateral ovariectomy before reaching the normal age of menopause is cardiovascular disease (CVD) [1]. While it has been established that hormone replacement therapy (HRT) reduces the prevalence of CVD in menopausal women [2], owing to the protective effect of oestrogen on the heart and vasculature $[3,4]$, a number of studies have demonstrated that HRT has a number of potential side effects [5] that may predispose them to breast and ovarian cancers $[6,7]$. Thus, more experimental work on the relationship between HRT and markers of CVD are warranted.
It is well established that lack of exercise is one of the main causes of CVD [8]. Exercise training improves health and disease status [8] by increasing cardiovascular fitness (CF) and strength, which make it a potent preventative tool for the aforementioned conditions, and it has shown positive effects on several types of cancer, dementia, or even recovery after spinal cord injury [8-10]. Exercise might also play an important role through menopause by attenuating the associated increased risk for CVD, osteoporosis, musculoskeletal symptoms, depression, and obesity $[9,11]$ by mimicking oestrogenic effects in early post-menopause $[12,13]$. However, relevant studies do not focus on the molecular level, intracellular signalling pathway apoptosis, or mediating physiological adaptations induced by acute and chronic exercise training. 
Recently several microRNAs (miRNA) have been described as playing an important role in CVD. miRNAs are small RNA sequences with the length of 20-24 nucleotides, which are able to suppress protein expression [14] and are recognised as mediators of exercise adaptation cardiac remodelling [15], skeletal muscle [16], and angiogenesis [17]. They are also involved in the prevention of atherosclerosis [18], and heart failure in ovariectomised rats [19]. However, little is known about exercise-induced modulation on miRNA biogenesis. Some studies have revealed that changes in the transcriptome homeostasis are critically associated with different processes in the heart [20]. The benefits of the miR-29 family in the development of cardiac [21], liver [22], kidney [23], and pulmonary [24] fibrosis have also been studied in an experimental model. Moreover, it has been described that the exercise training modify the expression levels of the different members of the miR-29 family under pathological conditions in experimental animal models. To our knowledge, no clinical studies have examined the effects resistance-endurance exercise (RE) on the cardiac microRNA-29 expression after menopause. Admittedly, such a study could be tenuous in humans. Therefore, the current study endeavours to elucidate the expression of microRNA-29 following RE in rats. The primary outcome for our study was microRNA-29 expression in ovariectomised (OVX) rats. We hypothesised that OVX would reduce cardiac gene microRNA-29 expression in heart muscle and that an RE training intervention would attenuate this response in ovariectomised rats.

\section{Material and methods}

\section{Animal model}

We have presented a schematic of the experimental design of the study in Figure 1. Thirty healthy female Wistar rats (weight: $200 \pm 25$ g; age: 30 weeks) were supplied by the Animal Research Centre, IAU, Tabriz Branch, Iran, and the University Ethics Committee approved all research procedures (2017/5200690). All animals received ethical treatment according to scientific standards $[25,26]$. All animals were housed on a $12: 12$ hour dark-light cycle at $22 \pm 1^{\circ} \mathrm{C}$ and $55 \pm 3 \%$ relative humidity and had free access to a standard chow and water ad libitum. After a one-week acclimation period, the rats were surgically ovariectomised as described previously [25] and continued to receive a basal diet during the week following surgery. Thereafter rats were randomly assigned to one of the following three treatment groups ( $n=10)$ : 1)SHAM rats incised and sutured without removal of the ovaries; 2 ) OVX rats following bilateral ovary removal; and 3) OVX + RE. Treatment started two weeks after surgery. The diets of all experimental animal were prepared according to modified American Institute of Nutrition (AIN)-76.

\section{Exercise training}

The exercise training combined the two endurance and resistance models as described previously [25]. Briefly, the exercise sessions were performed in a continuous sequence (RE training) for six weeks (five times per week). The first week of training was used as a run-in for $\mathrm{OVX}+\mathrm{RE}$ rats on the treadmill only to habituate rats to exercising on a motor-driven treadmill at $10 \mathrm{~m} / \mathrm{min}$, without an incline, for $11 \mathrm{~min} /$ day for five days, as described previously [25]. Following this period, all rats ran on the treadmill five days/week for six weeks. The running protocol was as follows: week $1=15 \mathrm{~m} / \mathrm{min}$ for $25 \mathrm{~min}$; week $2=16 \mathrm{~m} / \mathrm{min}$ for $32 \mathrm{~min}$; week $3=17 \mathrm{~m} / \mathrm{min}$ for $39 \mathrm{~min}$; week $4=18 \mathrm{~m} / \mathrm{min}$ for $46 \mathrm{~min}$; week $5=$ $53 \mathrm{~m} / \mathrm{min}$; and week $6=20 \mathrm{~m} / \mathrm{min}$ for $60 \mathrm{~min}$. Resistance training was performed for six weeks as previously described [26]. A ladder $(110 \mathrm{~cm}$ high, $18 \mathrm{~cm}$ wide, $2 \mathrm{~cm}$ space between steps) was placed at an $80^{\circ}$ inclination, and a resting chamber $(20 \mathrm{~cm} \times 20 \mathrm{~cm} \times 20 \mathrm{~cm})$ was placed at the top of the ladder. The rats climbed the ladder carrying resistance attached to the base of the tail, and the weights were gradually increased according to exercise training progression. Animals were familiarised with the resistance training apparatus by performing exercises during three consecutive days with nine repetitions per day. Resistance training sessions consisted of one set of climbing, using progressive loads, interspaced with 90-second intervals. The rats had to perform 8-10 repetitions to go from the base to the top of the ladder. The load was progressively increased from $50 \%$ of the maximum load in the first series, through $75 \%$ and $90 \%$, up to $100 \%$ of the maximum load in the fourth series. After that, the weight was increased in increments of $30 \mathrm{~g}$ per attempt until failure. Training was performed in the afternoon, five times a week, from Monday to Friday.

\section{Ovariectomy surgery}

Ovariectomised animals were anaesthetised with a solution of ketamine $(100 \mathrm{mg} / \mathrm{kg}$, i.p.) and xylazine $(10 \mathrm{mg} / \mathrm{kg}$, i.p.). Rats then received a mid-line dorsal skin incision approximately $3 \mathrm{~cm}$ long, and each ovary and part of the oviduct was removed. Following surgery, the muscles and the skin of surgical site were sutured, and the animals were kept warm. In the SHAM group, the same surgical procedures were performed as those for the OVX groups, but the ovaries were not removed.

\section{Molecular analysis}

\section{RNA isolation and cDNA synthesis}

Rats were sacrificed $24 \mathrm{~h}$ after the last exercise training session, and hearts were excised at the end of the six-week exercise training period. The method 


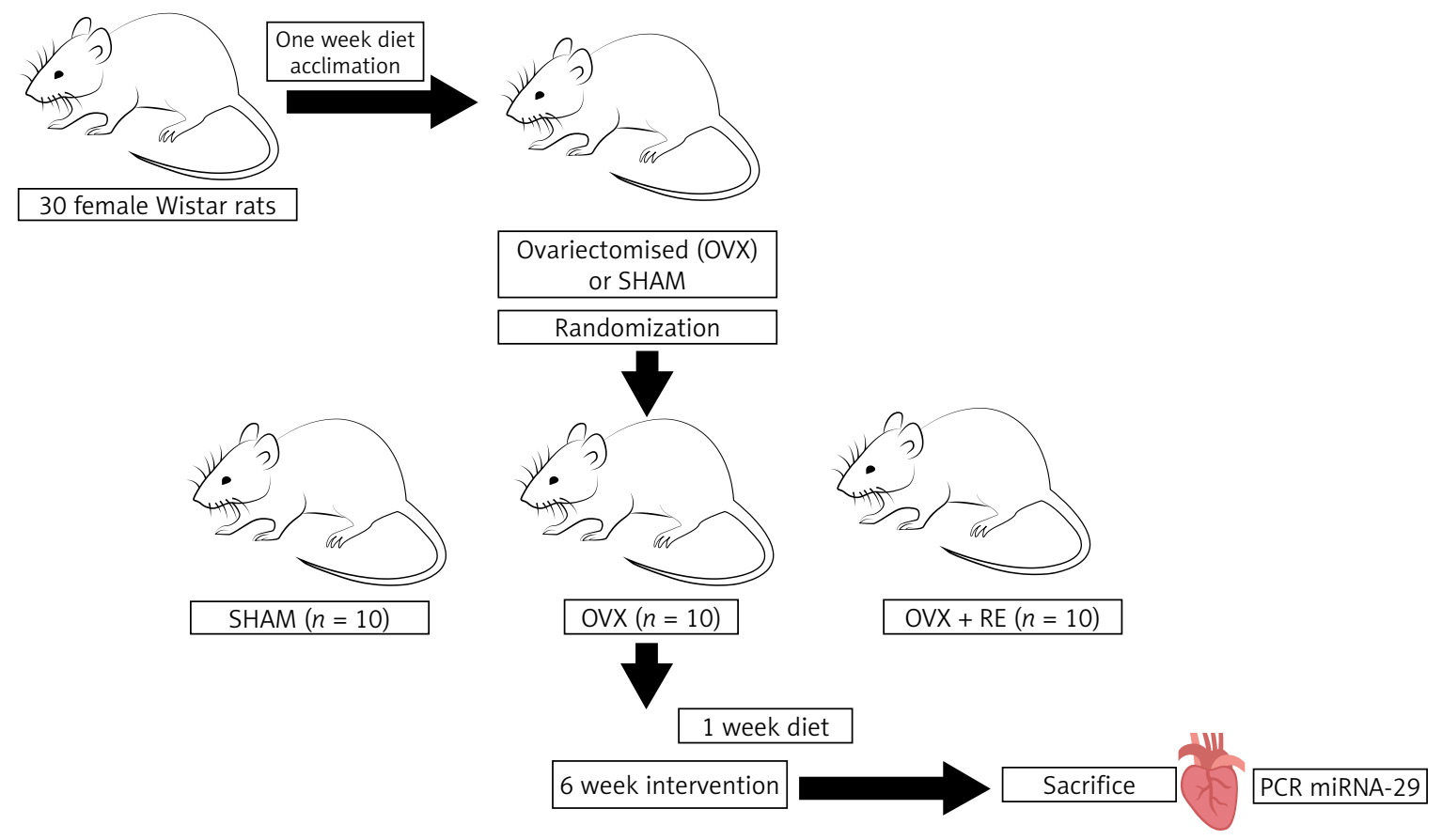

SHAM - sham-operated, OVX - ovariectomised, OVX + RE - ovariectomised with 6-weeks resistance-endurance exercise training

Fig. 1. Experimental design of the study

used for extraction of RNA and synthesis of cDNA was a modification of the methods previously described [27]. The total RNA was extracted from the left ventricle of the heart using a TRIzol RNX-Plus solution kit (Fermentase, Cinagen Co., Iran), according to the manufacturer's instructions. The cDNA from an RNA template was synthesised using total RNA and miScript II RT Kit (ParsGenome Co., Iran), following the manufacturer's instructions, and used as a template in PCR reactions for PCR primer pairs (Table 1). Real-time PCR was performed on a fast real-time PCR system (Fast Real Time PCR ABI 7500, Applied Biosystems, USA) using SYBR Green Kit (Exiqon, Qiagen, Denmark) and related samples. Hsa-RNU6B (U6) was used as an internal standard for miRNAs expression. Relative gene expression was performed by comparative cycle threshold $\left(\Delta \Delta C_{T}\right)$ method.

\section{Statistical analysis}

All statistical analyses were performed using SPSS 17 software, and significance was established at $p<0.05$. Changes in gene expression confirmed via real-time PCR and tests and treatment effects were analysed for significance using a two (time) by three (3) ANOVA and Tukey post-hoc assessment for between-group comparisons, as appropriate. Shapiro-Wilk tests were used to confirm the normal distribution of data. All data are presented as means \pm SEM.
Table 1. Primer sequences for each of the genes in the studied groups

\begin{tabular}{lcc}
\hline Primer of target & Sequence (5' to 3') ${ }^{*}$ & Base (bp) \\
\hline $\begin{array}{l}\text { microRNA-29 } \\
\text { forward }\end{array}$ & TGACTGGAGCATTAACCCTTGCA & 23 \\
\hline $\begin{array}{l}\text { microRNA-29 } \\
\text { reverse }\end{array}$ & TGTCCCATAAACGGCTCTGA & 20 \\
\hline U6 forward & CAAGATCATCAGCAATGCCTCC & 22 \\
\hline U6 reverse & GCCATCACGCCAGTTTCC & 18 \\
\hline *Sequences were derived from miRBase (www.mirbase.org) &
\end{tabular}

Table 2. Food intake, and body and heart weight of the rats during the study period

\begin{tabular}{lccc}
\hline Factor & SHAM & OVX & OVX + RE training \\
\hline$n$ & 10 & 10 & 10 \\
\hline BW final (g) & $264.1 \pm 2.6$ & $283.2 \pm 4.2^{*}$ & $263.7 \pm 3.5$ \\
\hline HW (mg) & $785.4 \pm 9.3$ & $808.1 \pm 11.1^{\star}$ & $821.3 \pm 10.6^{\text {** }}$ \\
\hline HW/BW (mg/g) & $2.9 \pm 2.16$ & $2.8 \pm 2.16$ & $3.11 \pm 2.16^{\text {** }}$ \\
\hline $\begin{array}{l}\text { Food intake } \\
\text { (g/day) }\end{array}$ & $16.43 \pm 0.11$ & $16.13 \pm 0.21$ & $15.84 \pm 0.13$ \\
\hline
\end{tabular}

BW - body weight, HW - heart weight, HW/BW - heart to body weight ratio, OVX - ovariectomised group, OVX + RE - ovariectomised with six-week RE training group; values are means $\pm \operatorname{SEM}(n=10),{ }^{*} p<0.05$ vs. SHAM. ${ }^{* *} p<0.05$ vs. SHAM \& OVX.

\section{Results}

Overall, we observed significant time, treatment, and time-by-treatment effects for body weight (BW), heart weight (HW), and mRNA-29 gene expression (all, 


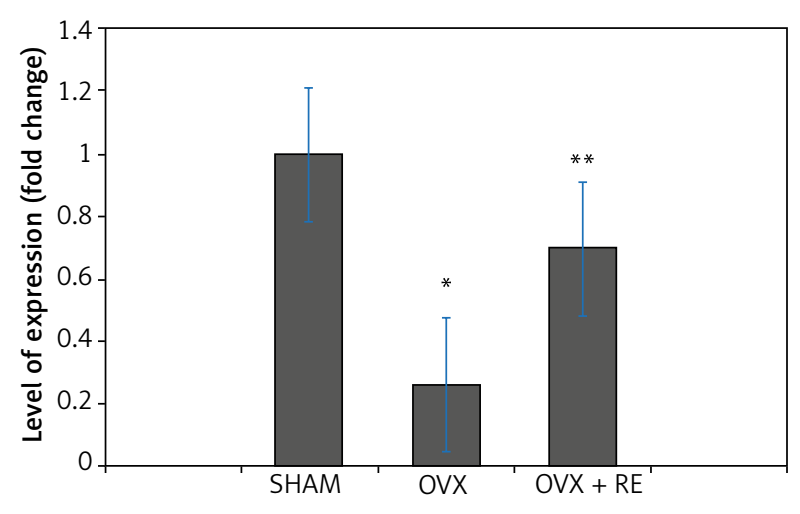

OVX - ovariectomised group, OVX + RE - ovariectomised with 6 weeks of resistance-endurance training group; "significant difference compare with SHAM group $(p<0.05)$, "*significant difference compared with the SHAM and OVX groups $(p<0.05)$; data are expressed as mean \pm SEM

Fig. 2. Level of microRNA-29 expression the hearts of the ovariectomised rats after treatment with resistance-endurance training

$p<0.05)$. Findings for $\mathrm{BW}, \mathrm{HW}, \mathrm{HW} / \mathrm{BW}$ ratio, and food intake are shown in Table 2. Weight gain was significantly greater in the OVX than the SHAM group six weeks after experiment $(p<0.05)$. Moreover, HW was significantly greater in the OVX + RE group than in the OVX and SHAM groups $(p<0.05)$. However, the HW/BW ratio increased more in OVX + RE than in the SHAM and OVX group $(p<0.05)$. There were no significant differences in daily food intake between the groups. Levels for the expression of the microRNA-29 are presented in Figure 2. Post-hoc analysis demonstrated that microRNA-29 expression was significantly lower in the OVX and OVX + RE group vs. the SHAM group ( $p<0.05)$; however, the OVX + RE group was significantly greater than the OVX group $(p<0.05)$.

\section{Discussion}

The primary aim of our study was to examine the effects of combined endurance and resistance training on the microRNA-29 gene expression in ovariectomised rats. We hypothesised that this combination of exercise would attenuate the ameliorated cardiac gene expression pattern observed in ovariectomised rats following surgery. The results of our study demonstrated that while microRNA-29 gene expression was reduced in all ovariectomised rats, exercise training attenuated this reduction in rats receiving an exercise intervention to an extent that was significantly different from that seen in OVX only. Therefore, we accept our research hypothesis. Our findings are of potential importance to women following menopause, which carries an increased risk of CVD [28, 29].

In this study, we used the ovariectomised rat as a model of natural menopause. There is evidence that physiological and metabolic changes by oestrogen de- ficiency due to OVX are similar to those in postmenopausal women [28]. The present study was designed to investigate the effects of RE training on the menopause-induced reduction of cardiac gene expression in a postmenopausal animal model. In the present study, we observed increased body and heart weight in OVX compared with the sham rats. We also demonstrated a significant increase in heart weight and heart-weight to body-weight ratio in the RE training group compared to the OVX group. Our results, in line with previous studies [25, 27, 30], demonstrate that increased weight gain, heart weight, LDL levels, and decreased HDL level after ovariectomy are reversed by exercise training.

Several studies have shown that microRNAs act in cardiac processes and play an important role in modulating some cardiovascular conditions, such as hypertrophy, myocardial ischaemia, endothelial dysfunction, cell death, and fibrosis [31-34]. MicroRNAs are also involved in the regulation of gene expression during heart functional alterations [33]. Interestingly, microRNA-29 expression levels seem to change in menopause [35].

Data from the present study indicate that ovariectomy decreased cardiac microRNA-29 gene expression in comparison with the sham surgery rats. These findings are in agreement with previous studies showing that ovariectomy decreases cardiac expression of miRNA-29, which may be associated with anti-fibrosis, which may in turn be associated with IGF-1 gene expression [30]. Previous experimental studies have shown that removal of oestrogen has a profound impact on myocardial gene expression, and oestrogen replacement can protect against negative changes associated with oestrogen removal [36]. Our finds are in line with these publications.

Further, after observing that OVX down-regulated expression of miRNA-29, we were interested in understanding whether cardiac miRNA-29 gene expression would be up-regulated with six weeks of regular RE training. Studies have shown that menopausal women have a higher chance of apoptotic and fibrosis death of cardiomyocytes contribution from CVD than menopausal women without CVD [37-39]. Generally, investigations have shown that OVX-associated increases in caspase 3, a key mediator of apoptotic and calpain, a calcium-dependent cysteine protease, suggesting an overall increase in the proapoptotic cardiac profile [35, 40]. Thus, it is possible that OVX in postmenopausal women may augment and increase the risk of heart disease.

Animal models suggest that exercise training modifies the expression levels of miR-29 under pathological conditions by influencing collagen pathways [41, 42]. To our knowledge, no study has been published so far uncovering the effects of RE training on the cardiac microRNA-29 expression in the heart of OVX rodents. Whilst our study was performed in rodents only, and 
measured only a marker of cardiac function (microRNA-29 expression), the data warrant the investigation of RE training prescription as a prevention strategy for the increased risk of CVD associated with menopause in woman.

Taken together, our findings support the hypothesis that six weeks of RE training can reverse the ovariectomy-induced changes in microRNA-29 expression in the heart of ovariectomised rats. This result suggests that RE training increase microRNA-29 expression levels probably by increasing microRNA-29 target gene IGF1 and anti-fibrosis associate genes [30]. Studies have shown that decreased microRNA-29 gene expression levels are related to a decline in IGF-1 gene expression, which leads to cardiac disorder in OVX [30, 43].

MicroRNA-29, an anti-fibrosis cardiac gene, regulates fibrosis signalling by increased IGF-1 formation and/or activation in both human [44 and animal [45] models. Habibi et al. [46] recently reported the upregulation of microRNA-29 with swimming training in OVX rats and suggested that swimming exercise training protectively regulates the heart against fibrosis by targeting the IGF-1 signalling pathway. These findings, combined with numerous reports on microRNA-29 gene as a fibrosis suppressor [15, 47], suggest that exercise training plays an important role in the development of cardiac fibrosis under pathological conditions. Nevertheless, the molecular and cellular adaptations in the cardiac microRNAs level are distinct according to the type of exercise in different animal models.

\section{Limitations}

There are several limitations associated with our study. First, we utilised an exercise-training program that added a resistance/overload component to endurance exercise. Hence, future trainings should examine the effects of our treatment using endurance- and resistance-only training models. Second, it is clear from our experimental model that we are unable to generalise our results to humans and, more specifically, women. However, our results are important because women present with cardiac inflammatory biomarkers a decade after men, which has been attributed to the beneficial effect of the female ovarian sex hormones that are lost after menopause [30]. Furthermore, animal and observational studies in humans have shown benefits of hormone replacement therapy when it is initiated early in the premenopausal [30] and postmenopausal period [25, 27]. Experimentally, ovariectomy reduces plasma oestradiol levels [31] and HDL cholesterol, whilst body weight, total cholesterol, LDL cholesterol, and triglyceride levels all increase in women after menopause [31, 32].

\section{Conclusions}

In conclusion, a regular RE training appears to be effective in inhibiting the development of cardiac risk factors associated with menopause. Future research should attempt to identify whether our observations on microRNA-29 expression translate into measurable physiological effects on vascular function, particularly in skeletal muscle. To our knowledge, this study is the first to assess the cardioprotective effect of six weeks of RE training on menopause-induced cardiac dysfunction in the heart of ovariectomised rats. These beneficial effects may be caused by enhancement of the cardiac function through inhibition of fibrosis by increased microRNA-29 expression.

\section{Disclosure}

The authors report no conflict of interest.

\section{REFERENCES}

1. Rivera CM, Grossardt BR, Rhodes DJ, et al. Increased cardiovascular mortality following early bilateral oophorectomy. Menopause 2009; 16 : 15-23.

2. Stampfer MJ, Colditz GA. Estrogen replacement therapy and coronary heart disease: a quantitative assessment of the epidemiologic evidence. Prev Med 1991; 20: 47-63.

3. Mendelsohn ME, Karas RH. The protective effects of estrogen on cardiovascular system. N Engl J Med 1999; 340: 1801-1111.

4. Scuteri A, Ferrucci L. Blood pressure, arterial function, structure, and aging: the role of hormonal replacement therapy in postmenopausal women. J Clin Hypertens 2003; 5: 219-225.

5. Rossouw JE, Anderson GL, Prentice RL, et al. Risks and benefits of estrogen plus progestin in healthy postmenopausal women: principal results from the Women's Health Initiative randomized controlled trial. JAMA 2002; 288: 321-333.

6. Yager JD, Davidson NE. Estrogen carcinogenesis in breast cancer. N Engl J Med 2006; 354: 270-282.

7. Burkman RT, Collins JA, Greene RA. Current perspectives on benefits and risks of hormone replacement therapy. Am J Obstet Gynecol 2001; 185: S13-S23.

8. Silva GJJ, Bye A, El Azzouzi H, Wisløff U. MicroRNAs as Important Regulators of Exercise Adaptation. Prog Cardiovasc Dis 2017; 60: 130-151.

9. Al-Nakkash L, Martin JB, Petty D, et al. Dietary genistein induces sexdependent effects on murine body weight, serum profiles, and vascular function of thoracic aortae. Gender Med 2012; 9: 295-308.

10. Moreira JB, Bechara LR, Bozi LH, et al. High- versus moderate-intensity aerobic exercise training effects on skeletal muscle of infarcted rats. J Appl Physiol 2013; 114: 1029-1041.

11. Messina M. Soy foods, isoflavones, and the health of postmenopausal women. Am J Clin Nutr 2014; 100 (Suppl): 423S-430S.

12. Maturana MA, Irigoyen MC, Spritzer PM. Menopause, estrogens, and endothelial dysfunction: current concepts. Clinics 2007; 62: 77-86.

13. Friedenreich CM. Physical activity and breast cancer risk: the effect of menopausal status. Exerc Sport Sci Rev 2004; 32: 180-184.

14. Lee RC, Feinbaum RL, Ambros V. The C. elegans heterochronic gene lin4 encodes small RNAs with antisense complementarity to lin-14. Cell 1993; 75: 843-854.

15. Soci UP, Fernandes T, Hashimoto NY, et al. MicroRNAs 29 are involved in the improvement of ventricular compliance promoted by aerobic exercise training in rats. Physiol Genomics 2011; 43: 665-673.

16. Davidsen PK, Gallagher IJ, Hartman JW, et al. High responders to resistance exercise training demonstrate differential regulation of skeletal muscle microRNA expression. J Appl Physiol 2011; 110: 309-317. 
17. Da Silva Jr ND, Fernandes T, Soci UP, et al. Swimming training in rats increases cardiac microRNA-126 expression and angiogenesis. Med Sci Sports Exerc 2012; 44: 1453-1462.

18. Radom-Aizik S, Zaldivar Jr FP, Haddad F, Cooper DM. Impact of brief exercise on circulating monocyte gene and microRNA expression: implications for atherosclerotic vascular disease. Brain Behav Immun 2014; 39: 121-129.

19. Van Eickels M, Grohé C, Cleutjens JP, et al. 17ß-Estradiol attenuates the development of pressure-overload hypertrophy. Circulation 2001; 104: 1419-1423.

20. Leptidis S, El Azzouzi H, Lok SI, et al. A deep sequencing approach to uncover the miRNOME in the human heart. PLoS One 2013; 8: e57800.

21. Van Rooij E, Sutherland LB, Thatcher JE, et al. Dysregulation of microRNAs after myocardial infarction reveals a role of miR-29 in cardiac fibrosis. Proc Natl Acad Sci U S A 2008; 105: 13027-13032.

22. Roderburg C, Urban GW, Bettermann K, et al. Micro-RNA profiling reveals a role for miR-29 in human and murine liver fibrosis. Hepatology 2011; 53: 209-218.

23. Wang B, Komers R, Carew R, et al. Suppression of microRNA-29 expression by TGF-beta1 promotes collagen expression and renal fibrosis J Am Soc Nephrol 2012; 23: 252-265.

24. Cushing L, Kuang PP, Qian J, et al. miR-29 is a major regulator of genes associated with pulmonary fibrosis. Am J Respir Cell Mol Biol 2011; 45: 287-294.

25. Jourkesh M, Choobineh S, Soori R, Ravasi AA. Effect of combined endurance-resistance training and soy extract supplementation on expression of eNOS gene in rats. Arch Med Sci Atheroscler Dis 2017; 2: e76-e81.

26. Oh HY, Lim S, Lee JM, et al. A combination of soy isoflavone supplementation and exercise improves lipid profiles and protects antioxidant defense-systems against exercise-induced oxidative stress in ovariectomized rats. Biofactors 2007; 29: 175-185.

27. Habibi P, Alihemmati AR, Nour Azar AR, et al. Expression of the Mir-133 and $\mathrm{BCl}-2$ could be affected by swimming training in the heart of ovariectomized rats. Iran J Basic Med Sci 2016; 19: 381-387.

28. Jin L, Hyung SC, Dae YK, et al. Combined effects of exercise and soy isoflavone diet on paraoxonase, nitric oxide and aortic apoptosis in ovariectomized rats. Appetite 2012; 58: 462-469.

29. Melton SA, Hegsted M, Keenan MJ, et al. Swimming eliminates the weight gain and abdominal fat associated with ovariectomy in the retired breeder rat despite high-fat diet selection. Appetite 2000; 35: 1-7.

30. Habibi P, Alihemmati AR, Nasirzadeh MR, et al. Involvement of microRNA-133 and -29 in cardiac disturbances in diabetic ovariectomized rats. Iran J Basic Med Sci 2016; 19: 1177-1185.

31. Hamilton KL, Lin L, Wang Y, Knowlton AA. Effect of ovariectomy on cardiac gene expression: inflammation and changes in SOCS gene expression. Physiol Genomics 2008; 32: 254-263.

32. Abbate A, Bussani R, Amin MS, et al. Acute myocardial infarction and heart failure: role of apoptosis. Int J Biochem Cell Biol 2006; 38: 18341840

33. Maric C. Risk factors for cardiovascular disease in women with diabetes. Gender Med 2010; 7: 551.

34. Liou CM, Yang AL, Kuo CH, et al. Effects of 17 beta-estradiol on cardiac apoptosis in ovariectomized rats. Cell Biochem Funct 2010; 28: 521528.

35. Webster KA, Graham RM, Thompson JW, et al. Bishopric NH. Redox stress and the contributions of $\mathrm{BH} 3$-only proteins to infarction. Antioxid Redox Signal 2006; 8: 1667-1676.

36. Ikeda S, Kong SW, Lu J, et al. Altered microRNA expression in human heart disease. Physiol Genomics 2007; 31: 367-373.

37. Thum T, Galuppo P, Wolf C, et al. MicroRNAs in the human heart: a clue to fetal gene reprogramming in heart failure. Circulation 2007; 116: 258-267.

38. Matkovich SJ, Van Booven DJ, Youker KA, et al. Reciprocal regulation of myocardial microRNAs and messenger RNA in human cardiomyopathy and reversal of the microRNA signature by biomechanical support. Circulation 2009; 119: 1263-1271.

39. Bonci D. MicroRNA-21 as therapeutic target in cancer and cardiovascular disease. Recent patents on cardiovascular drug discovery 2010; 5: 156-161.
40. Lacey JV, Mink Jr PJ, Lubin JH, et al. Menopausal hormone replacement therapy and risk of ovarian cancer. JAMA 2002; 288: 334-341.

41. Roncarati R, Viviani Anselmi C, Losi MA, et al. Circulating miR-29a, among other up-regulated microRNAs, is the only biomarker for both hypertrophy and fibrosis in patients with hypertrophic cardiomyopathy. J Am Coll Cardiol 2014; 63: 920-927.

42. Melo SF, Fernandes T, Barauna VG, et al. Expression of microRNA-29 and collagen in cardiac muscle after swimming training in myocardialinfarcted rats. Cell Physiol Biochem 2014; 33: 657-669.

43. Ban CR, Twigg SM. Fibrosis in diabetes complications: pathogenic mechanisms and circulating and urinary markers. Vasc Health Risk Manag 2008; 4: 575.

44. Neri Serneri GG, Boddi M, Modesti PA, et al. Increased cardiac sympathetic activity and insulin-like growth factor-I formation are associated with physiological hypertrophy in athletes. Circ Res 2001; 89: 977-982.

45. Fuller SJ, Mynett JR, Sugden PH. Stimulation of cardiac protein synthesis by insulin-like growth-factors. Biochem J 1992; 282: 85-90.

46. Habibi P, Alihemmatti A, Alipour M, et al. Effect of exercise on mir-29 and IGF-1 expression and lipid profile in the heart of ovariectomized rats. Acta Endocrinol 2016; 2: 130-136.

47. Van Rooij E, Sutherland LB, Thatcher JE, et al. Dysregulation of microRNAs after myocardial infarction reveals a role of miR-29 in cardiac fibrosis. Proc Natl Acad Sci U S A 2008; 105: 13027-13032. 